PROCEEDINGS OF THE

AMERICAN MATHEMATICAL SOCIETY

Volume 136, Number 8, August 2008, Pages 2959-2967

S 0002-9939(08)09445-8

Article electronically published on March 14, 2008

\title{
STATISTICS ON RIEMANNIAN MANIFOLDS: ASYMPTOTIC DISTRIBUTION AND CURVATURE
}

\author{
ABHISHEK BHATTACHARYA AND RABI BHATTACHARYA
}

(Communicated by Edward C. Waymire)

\begin{abstract}
In this article a nonsingular asymptotic distribution is derived for a broad class of underlying distributions on a Riemannian manifold in relation to its curvature. Also, the asymptotic dispersion is explicitly related to curvature. These results are applied and further strengthened for the planar shape space of k-ads.
\end{abstract}

\section{INTRODUCTION}

Statistical analysis of a probability measure $Q$ on a differentiable manifold $M$ has diverse applications in directional and axial statistics, morphometrics, medical diagnostics and machine vision $(1,1,2,3,4,6,17,8,11,15)$. Most of this analysis focuses on nonintrinsic Fréchet means of $Q$. In this article we provide a distribution theory for the nonparametric analysis of intrinsic means which can be directly used for the one- and two-sample problems. To be precise, let $(M, g)$ be a Riemannian manifold with metric tensor $g$ and geodesic distance $d_{g}$. Define the Fréchet function $F$ of $Q$ as

$$
F(p)=\int_{M} d_{g}^{2}(p, m) Q(d m), p \in M .
$$

Assume $F$ to be finite. We consider probability measures $Q$ whose support $\operatorname{supp}(Q)$ are contained in geodesic balls $B(p, r)=\left\{m: d_{g}(p, m)<r\right\}$. If the Fréchet function, restricted to such a ball $B(p, r)$, has a unique minimizer $\mu_{I}$ in $B(p, r)$, we call it the intrinsic mean of $Q$ in $B(p, r)$. The sample intrinsic mean $\mu_{n I}$ in $B(p, r)$ is the intrinsic mean of $Q_{n}=\frac{1}{n} \sum_{j=1}^{n} \delta_{X_{j}}$ in $B(p, r)$, where $X_{1}, X_{2}, \ldots, X_{n}$ are independent and identically distributed (iid) observations from the underlying distribution $Q$. Crucial to nonparametric analysis is the asymptotic distribution of $\mu_{n I}$. Our main goals are (i) to derive this asymptotic distribution, assuring its nonsingularity, under as broad a condition on $\operatorname{supp}(Q)$ as possible, (ii) to explicitly compute the asymptotic dispersion, and (iii) to apply and refine the general theory to the particularly important planar shape space $\Sigma_{2}^{k}$ of $k$ landmarks introduced by Kendall 11 .

Received by the editors July 15, 2007.

2000 Mathematics Subject Classification. Primary 62G20; Secondary 62E20, 62H35.

Key words and phrases. Intrinsic mean, shape space of k-ads, nonparametric analysis.

This research was supported by NSF Grant DMS 04-06143.

(C)2008 American Mathematical Society Reverts to public domain 28 years from publication 
To indicate the role curvature plays in this endeavor, let $r_{*}=\min \left\{\operatorname{inj}(M), \frac{\pi}{\sqrt{\bar{C}}}\right\}$, where $\bar{C}$ is an upper bound of sectional curvatures of $M$ if this upper bound is positive, and $\bar{C}=0$ otherwise. Also, $\operatorname{inj}(M) \equiv \inf \left\{d_{g}(p, C(p)): p \in M\right\}$ is the injectivity radius of $M$, where $C(p)$ is the cut locus of $p$, i.e., the set of points of the form $\gamma\left(t_{0}\right)$, where $\gamma$ is a geodesic and $t_{0}$ is the supremum of all $t>0$ such that the geodesic from $p$ to $\gamma(t)$ is distance minimizing. The exponential map $\exp _{p}$ is injective on $\left\{v \in T_{p}(M):|v|<r\right\}$ if and only if $r \leq r_{*}$ (Do Carmo [5], p. 271). It follows that a geodesic ball $B(p, r)$ with $r \leq \frac{r_{*}}{2}$ is strongly convex, i.e., for every pair $q, q^{\prime} \in B(p, r)$ there exists a unique geodesic connecting $q, q^{\prime}$, entirely contained in $B(p, r)$, this geodesic being distance minimizing. By Proposition 2.1 and Theorem 2.2, if $\operatorname{supp}(Q) \subseteq B\left(p, \frac{r_{*}}{2}\right)$, then $Q$ has a unique intrinsic mean $\mu_{I}$ in $B\left(p, \frac{r_{*}}{2}\right)$. If in addition, $\operatorname{supp}(Q) \subseteq B\left(\mu_{I}, \frac{r_{*}}{2}\right)$, then the sample intrinsic mean has asymptotic normal distribution. Further, in the case of manifolds with constant sectional curvature, the asymptotic dispersion can be explicitly expressed in terms of curvature.

It may be noted that our results are not related to those of Pennec [16 who has a number of interesting results on distributions on manifolds, including one that provides an expansion of the density of the (analog of) normal distribution on the manifold in terms of its variance, for the case of small variance.

For background in differential geometry used here, we refer to Do Carmo [5] and Lee [14.

\section{Asymptotic Distribution and CURVATURe}

Let $(M, g)$ be a Riemannian manifold. We continue to use the notation of Section 1 . Let $Q$ be a probability measure, $\sup (Q) \subseteq B\left(p, \frac{r_{*}}{2}\right)$ for some $p$. Then there is a unique (local) intrinsic mean $\mu_{I}$ in $B\left(p, \frac{r_{*}}{2}\right)$ (Kendall [12]). This substantially extends Karchar's result on the existence and uniqueness of a local mean (9]), but not his important result on the strict convexity of $F$. We are able to circumvent this difficulty in the case $\operatorname{supp}(Q) \subseteq B\left(\mu_{I}, \frac{r_{*}}{2}\right)$. Denote by $\mu_{n I}$ the intrinsic mean of $Q_{n}$ in $B\left(p, \frac{r_{*}}{2}\right)$. The inverse of the exponential map $\phi=\exp _{p}^{-1}$ is a diffeomorphism on $B\left(p, \frac{r_{*}}{2}\right)$ onto its image, say $U$, in $T_{p}(M)$. The image $\tilde{Q}=Q \circ \phi^{-1}$ of $Q$ under $\phi$ is a probability measure in $T_{p}(M)$, and the image $\mu=\phi\left(\mu_{I}\right)$ of $\mu_{I}$ is the minimizer of

$$
\tilde{F}(x)=\int_{U} d_{g}^{2}\left(\phi^{-1} x, \phi^{-1} y\right) \tilde{Q}(d y), x \in U .
$$

Similarly $\mu_{n}=\phi\left(\mu_{n I}\right)$ is the corresponding minimizer when $\tilde{Q}$ is replaced by $\tilde{Q}_{n}=$ $\frac{1}{n} \sum_{j=1}^{n} \delta_{\phi\left(X_{j}\right)}$. As proved in [3], Theorem 2.1, a central limit theorem for the Mestimator $\mu_{n}$ may be derived and used to obtain the following result. The normal coordinates $x, y$ used here are with respect to a chosen orthonormal basis in $T_{p} M$.

Proposition 2.1. Suppose the support of $Q$ is contained in the geodesic ball $B=$ $B\left(p, \frac{r_{*}}{2}\right)$. Let $\phi=\exp _{p}^{-1}: B \longrightarrow \phi(B)$. Define $h(x, y)=d_{g}^{2}\left(\phi^{-1} x, \phi^{-1} y\right) ; x, y \in$ $\phi(B)$. Let $\left(\left(D_{r} h\right)\right)_{r=1}^{d}$ and $\left(\left(D_{r} D_{s} h\right)\right)_{r, s=1}^{d}$ be the matrices of first and second order derivatives of $y \mapsto h(x, y)$. Let $\tilde{X}_{j}=\phi\left(X_{j}\right) ; j=1, \ldots, n, X_{1}, \ldots, X_{n}$ being iid observations from $Q$. Define

$$
\Lambda=E\left(\left(D_{r} D_{s} h\left(\tilde{X}_{1}, \mu\right)\right)\right)_{r, s=1}^{d}, \Sigma=\operatorname{Cov}\left(\left(D_{r} h\left(\tilde{X}_{1}, \mu\right)\right)\right)_{r=1}^{d} .
$$


If $\Lambda$ is nonsingular, then

$$
\sqrt{n}\left(\mu_{n}-\mu\right) \stackrel{\mathcal{L}}{\longrightarrow} N\left(0, \Lambda^{-1} \Sigma \Lambda^{-1}\right) .
$$

The natural candidate for $p$ in Proposition 2.1 is the intrinsic mean of $Q$ in $B\left(p, \frac{r_{*}}{2}\right)$, namely $\mu_{I}$. Then we get expressions for $\Lambda$ and $\Sigma$ using an orthonormal basis in $T_{\mu_{I}} M$. Theorem 2.2 below gives a lower bound on $\Lambda$ and an exact expression when $M$ has constant sectional curvature. The lower bound gives a condition on the nonsingularity of $\Lambda$. The nonsingularity of $\Sigma$ is a milder condition which holds, for example, when $Q$ has a density with respect to the volume measure on $M$. In the statement of the theorem, the usual partial order $A \geq B$ between $d \times d$ symmetric matrices $A, B$, means that $A-B$ is nonnegative definite.

Theorem 2.2. Assume $\operatorname{supp}(Q) \subseteq B\left(p, \frac{r_{*}}{2}\right)$. Let $\phi=\exp _{\mu_{I}}^{-1}: B\left(p, \frac{r_{*}}{2}\right) \longrightarrow$ $T_{\mu_{I}} M\left(\approx \mathbb{R}^{d}\right)$, and let $C$ denote an upper bound of all sectional curvatures. Then in normal coordinates with respect to a chosen orthonormal basis in $T_{\mu_{I}} M$,

$$
\begin{aligned}
& D_{r} h(x, 0)=-2 x^{r}, \quad 1 \leq r \leq d, \\
& {\left[D_{r} D_{s} h(x, 0)\right] \geq\left[2\left(\left(\frac{1-f(|x|)}{|x|^{2}}\right) x^{r} x^{s}+f(|x|) \delta_{r s}\right)\right]_{1 \leq r, s \leq d},} \\
& \text { where }|x|=\sqrt{\left(x^{1}\right)^{2}+\left(x^{2}\right)^{2}+\ldots+\left(x^{d}\right)^{2},} \\
& f(x)= \begin{cases}1 & \text { if } C=0, \\
\sqrt{C} x \frac{\cos (\sqrt{C} x)}{\sin (\sqrt{C} x)} & \text { if } C>0, \\
\sqrt{-C} x \frac{\cosh (\sqrt{-C} x)}{\sinh (\sqrt{-C} x)} & \text { if } C<0 .\end{cases}
\end{aligned}
$$

There is equality in (2.4) when $M$ has constant sectional curvature $C$, and in this case $\Lambda$ has the expression

$$
\Lambda_{r s}=2 E\left(\left(\frac{1-f\left(\left|\tilde{X}_{1}\right|\right)}{\left|\tilde{X}_{1}\right|^{2}}\right) \tilde{X}_{1}^{r} \tilde{X}_{1}^{s}+\left(f\left(\left|\tilde{X}_{1}\right|\right)\right) \delta_{r s}\right), \quad 1 \leq r, s \leq d,
$$

$\Lambda$ being positive definite if $Q$ has support in $B\left(\mu_{I}, \frac{r_{*}}{2}\right)$.

Proof. Let $\gamma(s)$ be a geodesic, $\gamma(0)=\mu_{I}$. Define $c(s, t)=\exp _{m}\left(\operatorname{texp}_{m}^{-1} \gamma(s)\right)$, $s \in[0, \epsilon], t \in[0,1]$, as a smooth variation of $\gamma$ through geodesics lying entirely in $B\left(p, \frac{r_{*}}{2}\right)$. Let $T=\frac{\partial}{\partial t} c(s, t), S=\frac{\partial}{\partial s} c(s, t)$. Since $c(s, 0)=m, S(s, 0)=0$, and since $c(s, 1)=\gamma(s), S(s, 1)=\dot{\gamma}(s)$. Also $\langle T, T\rangle=d_{g}^{2}(\gamma(s), m)$ is independent of $t$, and the covariant derivative $D_{t} T$ vanishes because $t \mapsto c(s, t)$ is a geodesic (for each $s$ ). Then

$$
d_{g}^{2}(\gamma(s), m)=\langle T(s, t), T(s, t)\rangle=\int_{0}^{1}\langle T(s, t), T(s, t)\rangle d t .
$$

Hence $d_{g}^{2}(\gamma(s), m)$ is $C^{\infty}$ smooth, and using the symmetry of the connection on a parametrized surface (see Lemma 3.4, p. 68 in Do Carmo [5]), we get

$$
\begin{aligned}
\frac{d}{d s} d_{g}^{2}(\gamma(s), m) & =2 \int_{0}^{1}\left\langle D_{s} T, T\right\rangle d t=2 \int_{0}^{1} \frac{d}{d t}\langle T, S\rangle d t \\
& =2\langle T(s, 1), S(s, 1)\rangle=-2\left\langle\exp _{\gamma(s)}^{-1} m, \dot{\gamma}(s)\right\rangle .
\end{aligned}
$$


Substituting $s=0$ in $(2.8)$, we get expressions for $D_{r} h(x, 0)$ as in (2.3). Also

$$
\begin{aligned}
\frac{d^{2}}{d s^{2}} d_{g}^{2}(\gamma(s), m) & =2\left\langle D_{s} T(s, 1), S(s, 1)\right\rangle \\
& =2\left\langle D_{t} S(s, 1), S(s, 1)\right\rangle=2\left\langle D_{t} J_{s}(1), J_{s}(1)\right\rangle,
\end{aligned}
$$

where $J_{s}(t)=S(s, t)$. Note that $J_{s}$ is a Jacobi field along $c(s,$.$) with J_{s}(0)=0$, $J_{s}(1)=\dot{\gamma}(s)$. Let $J_{s}^{\perp}$ and $J_{s}^{-}$be the normal and tangential components of $J_{s}$. The relations (2.13)-(2.15) below may be obtained from Jost [10], p. 197, and Lee [14, Lemma 10.8. For the sake of exposition, we indicate the arguements here. Let $\eta$ be a unit speed geodesic in $M$ and $J$ a normal Jacobi field along $\eta, J(0)=0$. Define

$$
u(t)= \begin{cases}t & \text { if } C=0, \\ \frac{\sin (\sqrt{C} t)}{\sqrt{C}} & \text { if } C>0, \\ \frac{\sinh (\sqrt{-C} t)}{\sqrt{-C}} & \text { if } C<0 .\end{cases}
$$

Then $u^{\prime \prime}(t)=-C u(t)$ and

$$
\left(|J|^{\prime} u-|J| u^{\prime}\right)^{\prime}(t)=\left(|J|^{\prime \prime}+C|J|\right) u(t) .
$$

By exact differentiation and Schwartz inequality, it is easy to show that $|J|^{\prime \prime}+$ $C|J| \geq 0$, hence $\left(|J|^{\prime} u-|J| u^{\prime}\right)^{\prime}(t) \geq 0$ whenever $u(t) \geq 0$. This implies that $|J|^{\prime} u-|J| u^{\prime} \geq 0$ if $t \leq t_{0}$, where $u$ is positive on $\left(0, t_{0}\right)$. Also $|J|^{\prime}=\frac{\left\langle J^{\prime}, J\right\rangle}{|J|}$. Therefore $\left\langle J(t), D_{t} J(t)\right\rangle \geq \frac{u^{\prime}(t)}{u(t)}|J(t)|^{2} \forall t<t_{0}$. If we drop the unit speed assumption on $\eta$, we get

$$
\left\langle J(1), D_{t} J(1)\right\rangle \geq|\dot{\eta}| \frac{u^{\prime}(|\dot{\eta}|)}{u(|\dot{\eta}|)}|J(1)|^{2} \text { if }|\dot{\eta}|<t_{0} .
$$

Here $t_{0}=\infty$ if $C \leq 0$ and equals $\frac{\pi}{\sqrt{C}}$ if $C>0$. When $M$ has constant sectional curvature $C, J(t)=u(t) E(t)$, where $E$ is a parallel normal vector field along $\eta$. Hence

$$
\left\langle J(t), D_{t} J(t)\right\rangle=u(t) u^{\prime}(t)|E(t)|^{2}=\frac{u^{\prime}(t)}{u(t)}|J(t)|^{2} .
$$

If we drop the unit speed assumption, we get

$$
\left\langle J(t), D_{t} J(t)\right\rangle=|\dot{\eta}| \frac{u^{\prime}(|\dot{\eta}| t)}{u(|\dot{\eta}| t)}|J(t)|^{2} .
$$

Since $J_{s}^{\perp}$ is a normal Jacobi field along the geodesic $c(s,$.$) , from (2.13)$ and $(2.15)$ it follows that

$$
\left\langle J_{s}^{\perp}(1), D_{t} J_{s}^{\perp}(1)\right\rangle \geq f(d(\gamma(s), m))\left|J_{s}^{\perp}(1)\right|^{2}
$$

with equality in (2.16) when $M$ has constant sectional curvature $C, f$ being defined in $(2.5)$.

Next suppose $J$ is a Jacobi field along a geodesic $\eta, J(0)=0$ and let $J^{-}(t)$ be its tangential component. Then $J^{-}(t)=\lambda t \dot{\eta}(t)$ where $\lambda t=\frac{\langle J(t), \dot{\eta}(t)\rangle}{|\dot{\eta}|^{2}}, \lambda$ being independent of $t$. Hence

$$
\begin{aligned}
\left(D_{t} J\right)^{-}(t) & =\frac{\left\langle D_{t} J(t), \dot{\eta}(t)\right\rangle}{|\dot{\eta}|^{2}} \dot{\eta}(t) \\
& =\frac{d}{d t}\left(\frac{\langle J(t), \dot{\eta}(t)\rangle}{|\eta|^{2}}\right) \dot{\eta}(t)=\lambda \dot{\eta}(t)=D_{t}\left(J^{-}\right)(t)
\end{aligned}
$$


and

$$
\begin{aligned}
D_{t}\left|J^{-}\right|^{2}(1) & =2 \lambda^{2}|\dot{\eta}|^{2}=2 \frac{\langle J(1), \dot{\eta}(1)\rangle^{2}}{|\dot{\eta}(1)|^{2}} \\
& =D_{t}\left\langle J, J^{-}\right\rangle(1)=\left\langle D_{t} J(1), J^{-}(1)\right\rangle+\left|J^{-}(1)\right|^{2}
\end{aligned}
$$

which implies

$$
\left\langle D_{t} J(1), J^{-}(1)\right\rangle=2 \frac{\langle J(1), \dot{\eta}(1)\rangle^{2}}{|\dot{\eta}(1)|^{2}}-\left|J^{-}(1)\right|^{2}=\frac{\langle J(1), \dot{\eta}(1)\rangle^{2}}{|\dot{\eta}(1)|^{2}} .
$$

Apply (2.17) and (2.18) to the Jacobi field $J_{s}$ to get

$$
\begin{aligned}
& D_{t}\left(J_{s}^{-}\right)(1)=\left(D_{t} J_{s}\right)^{-}(1)=J_{s}^{-}(1)=\frac{\left\langle J_{s}(1), T(s, 1)\right\rangle}{|T(s, 1)|^{2}} T(s, 1), \\
& \left\langle D_{t} J_{s}(1), J_{s}^{-}(1)\right\rangle=\frac{\left\langle J_{s}(1), T(s, 1)\right\rangle^{2}}{|T(s, 1)|^{2}} .
\end{aligned}
$$

Using (2.16), (2.19) and (2.20), (2.10) becomes

$$
\begin{aligned}
\frac{d^{2}}{d s^{2}} d_{g}^{2}(\gamma(s), m)= & 2\left\langle D_{t} J_{s}(1), J_{s}(1)\right\rangle \\
= & 2\left\langle D_{t} J_{s}(1), J_{s}^{-}(1)\right\rangle+2\left\langle D_{t} J_{s}(1), J_{s}^{\perp}(1)\right\rangle \\
= & 2\left\langle D_{t} J_{s}(1), J_{s}^{-}(1)\right\rangle+2\left\langle D_{t}\left(J_{s}^{\perp}\right)(1), J_{s}^{\perp}(1)\right\rangle \\
& \geq 2 \frac{\left\langle J_{s}(1), T(s, 1)\right\rangle^{2}}{|T(s, 1)|^{2}}+2 f(|T(s, 1)|)\left|J_{s}^{\perp}(1)\right|^{2} \\
= & 2 \frac{\left\langle J_{s}(1), T(s, 1)\right\rangle^{2}}{|T(s, 1)|^{2}}+2 f(|T(s, 1)|)\left|J_{s}(1)\right|^{2} \\
& -2 f(|T(s, 1)|) \frac{\left\langle J_{s}(1), T(s, 1)\right\rangle^{2}}{|T(s, 1)|^{2}} \\
& =2 f\left(d_{g}(\gamma(s), m)\right)|\dot{\gamma}(s)|^{2}+2\left(1-f\left(d_{g}(\gamma(s), m)\right) \frac{\left\langle\dot{\gamma}(s), e x p_{\gamma(s)}^{-1} m\right\rangle^{2}}{d_{g}^{2}(\gamma(s), m)}\right.
\end{aligned}
$$

with equality in (2.21) when $M$ has constant sectional curvature $C$. Substituting $s=0$ in $(2.22)$, we get a lower bound for $\left[D_{r} D_{s} h(x, 0)\right]$ as in $(2.4)$ and an exact expression for $D_{r} D_{s} h(x, 0)$ when $M$ has constant sectional curvature. To see this, let $\dot{\gamma}(0)=v$. Then writing $m=\phi^{-1}(x), \gamma(s)=\phi^{-1}(s v)$, one has

$$
\begin{aligned}
& \left.\frac{d^{2}}{d s^{2}} d_{g}^{2}(\gamma(s), m)\right|_{s=0}=\left.\frac{d^{2}}{d s^{2}} d_{g}^{2}\left(\phi^{-1}(x), \phi^{-1}(s v)\right)\right|_{s=0} \\
= & \left.\frac{d^{2}}{d s^{2}} h(x, s v)\right|_{s=0}=\sum_{r, s=1}^{d} v_{r} v_{s} D_{r} D_{s} h(x, 0) .
\end{aligned}
$$

Since $d^{2}(\gamma(s), m)$ is twice continuously differentiable and $Q$ has compact support, using the Lebesgue DCT, we get

$$
\left.\frac{d^{2}}{d s^{2}} F(\gamma(s))\right|_{s=0}=\left.\int \frac{d^{2}}{d s^{2}} d^{2}(\gamma(s), m)\right|_{s=0} Q(d m) .
$$

Then (2.6) follows from (2.22). If $\operatorname{supp}(Q) \subseteq B\left(\mu_{I}, \frac{r_{*}}{2}\right)$, then the expression in (2.22) is strictly positive at $s=0$ for all $m \in \operatorname{supp}(Q)$, hence $\Lambda$ is positive definite. This completes the proof. 
Under the assumptions of Theorem 2.2, it follows that $E\left(\tilde{X}_{1}\right)=0$ and $\Sigma=$ $4 E\left(\tilde{X}_{1} \tilde{X}_{1}^{\prime}\right)$. This is also stated in Theorem 2.1 in Bhattacharya [2].

Remark 2.1. It may be noted that the spaces $S^{d}, \mathbb{R} P^{d}$ have constant positive curvature. One may also endow the projective shape space with a metric which makes it a space of constant positive curvature, since it is diffeomorphic to a product of real projective spaces ([15]). In the next section we turn to $\Sigma_{2}^{k}$, whose sectional curvatures range from 1 to 4 .

\section{Application to the planar shape Space $\Sigma_{2}^{k}$}

Consider the planar shape space $\Sigma_{2}^{k}$ of $\mathbf{k - a d s}$ in $\mathbb{R}^{2}$. An element of $\Sigma_{2}^{k}$ is a set of $\mathrm{k}$ landmarks, or points in the plane (not all equal), modulo translation, rotation and scaling. Let $S_{2}^{k}$ be the pre-shape sphere which is the space of column vectors in $\mathbb{C}^{k}$ with mean 0 and norm 1 . Its tangent space is

$$
T_{z} S_{2}^{k}=\left\{v \in \mathbb{C}^{k}: v^{\prime} \mathbf{1}_{k}=\operatorname{Re}\left(z^{\prime} \bar{v}\right)=0\right\} .
$$

Here $\mathbf{1}_{k}$ denotes the column vector of ones of size $k$. To apply Proposition 2.1 to carry out nonparametric inference on $\Sigma_{2}^{k}$, we need to identify the exponential and inverse exponential maps on $\Sigma_{2}^{k}$. For that we consider their lifts to $S_{2}^{k}$ as in Section 4 in Le [13, and Kendall [11]. The map $\pi: S_{2}^{k} \rightarrow \Sigma_{2}^{k}$,

$$
z \mapsto \pi(z)=[z]=\{\lambda z: \lambda \in \mathbb{C},|\lambda|=1\},
$$

is a Riemannian submersion. So the tangent space $T_{[z]} \Sigma_{2}^{k}$ is isometric with a subspace of $T_{z} S_{2}^{k}$ called the horizontal subspace $H_{z}$ which is

$$
H_{z}=\left\{v \in \mathbb{C}^{k}: z^{\prime} \bar{v}=0, v^{\prime} \mathbf{1}_{k}=0\right\} .
$$

Denote the corresponding isometric mapping by $\chi_{[z]}: T_{[z]} \Sigma_{2}^{k} \rightarrow H_{z}$. Then $\exp _{[z]}=$ $\pi \circ \exp _{z} \circ \chi_{[z]}$, and

$$
\begin{aligned}
& \chi_{[z]} \circ \exp _{[z]}^{-1}: \Sigma_{2}^{k} \backslash C([z]) \rightarrow H_{z},[w] \mapsto \frac{r}{\operatorname{sinr}}\left\{-z \cos r+e^{i \theta} w\right\}, \\
& r=d_{g}([z],[w])=\arccos \left(\left|z^{\prime} \bar{w}\right|\right) \in\left[0, \frac{\pi}{2}\right), e^{i \theta}=\frac{z^{\prime} \bar{w}}{\left|z^{\prime} \bar{w}\right|} .
\end{aligned}
$$

In $(3.1), C([z])$ is the cut-locus of $[z]$, which is

$$
C([z])=\left\{[x] \in \Sigma_{2}^{k}: d_{g}([x],[z])=\frac{\pi}{2}\right\}=\left\{[x]: z^{\prime} \bar{x}=0\right\} .
$$

$\Sigma_{2}^{k}$ has all sectional curvatures bounded between 1 and 4 , and its injectivity radius is $\frac{\pi}{2}$. From a result due to Kendall [12, $Q$ has an intrinsic mean if its support is contained in a geodesic ball of radius $\frac{\pi}{4}$. Suppose $\operatorname{supp}(Q) \subseteq B\left(p, \frac{\pi}{4}\right)$ and let $\mu_{I}=[\mu]$ be the intrinsic mean of $Q$ in the support, with $\mu$ being one of its preshapes. The following theorem gives the expression for $\Lambda$ in Theorem 2.2 and derives a sufficient condition for its nonsingularity.

Theorem 3.1. Let $\phi: B\left(p, \frac{\pi}{4}\right) \rightarrow \mathbb{C}^{k-2}\left(\approx \mathbb{R}^{2 k-4}\right)$ be the coodinates of $\chi_{\mu_{I}}$ o $\exp _{\mu_{I}}^{-1}$ : $B\left(p, \frac{\pi}{4}\right) \rightarrow H_{\mu}$ with respect to some orthonormal basis $\left\{v_{1}, \ldots, v_{k-2}, i v_{1}, \ldots, i v_{k-2}\right\}$ for $H_{\mu}$. Define $h(x, y)=d_{g}^{2}\left(\phi^{-1} x, \phi^{-1} y\right)$. Let $\left(\left(D_{r} h\right)\right)_{r=1}^{2 k-4}$ and $\left(\left(D_{r} D_{s} h\right)\right)_{r, s=1}^{2 k-4}$ be the matrix of first and second order derivatives of $y \mapsto h(x, y)$. Let $\tilde{X}_{j}=\phi\left(X_{j}\right)=$ $\left(\tilde{X}_{j}^{1}, \ldots, \tilde{X}_{j}^{k-2}\right)^{\prime} ; j=1, \ldots, n, X_{1}, \ldots, X_{n}$ being iid observations from $Q$. Define 
$\Lambda=E\left(\left(D_{r} D_{s} h\left(\tilde{X}_{1}, 0\right)\right)\right)_{r, s=1}^{2 k-4}$. Then $\Lambda$ is positive definite if the support of $Q$ is contained in $B\left(\mu_{I}, R\right)$, where $R$ is the unique solution of $\tan (x)=2 x, x \in\left(0, \frac{\pi}{2}\right)$.

Proof. For a geodesic $\gamma$ starting at $\mu_{I}$, write $\gamma=\pi \circ \tilde{\gamma}$, where $\tilde{\gamma}$ is a geodesic in $S_{2}^{k}$ starting at $\mu$. From the proof of Theorem 2.2, for $m=[z] \in B\left(p, \frac{\pi}{4}\right)$,

$$
\begin{gathered}
\frac{d}{d s} d_{g}^{2}(\gamma(s), m)=2\langle T(s, 1), \dot{\gamma}(s)\rangle=2\langle\tilde{T}(s, 1), \dot{\tilde{\gamma}}(s)\rangle, \\
\frac{d^{2}}{d s^{2}} d_{g}^{2}(\gamma(s), m)=2\left\langle D_{s} T(s, 1), \dot{\gamma}(s)\right\rangle=2\left\langle D_{s} \tilde{T}(s, 1), \dot{\tilde{\gamma}}(s)\right\rangle,
\end{gathered}
$$

where $\tilde{T}(s, 1)=\chi_{\gamma(s)}(T(s, 1))$. From (3.1), this has the expression

$$
\begin{aligned}
\tilde{T}(s, 1) & =-\frac{\rho(s)}{\sin (\rho(s))}\left[-\cos (\rho(s)) \tilde{\gamma}(s)+e^{i \theta(s)} z\right], \\
\text { where } e^{i \theta(s)} & =\frac{\bar{z}^{\prime} \tilde{\gamma}(s)}{\cos (\rho(s))}, \rho(s)=d_{g}(\gamma(s), m) .
\end{aligned}
$$

The inner product in (3.3) and (3.4) is the Riemannian metric on $T S_{2}^{k}$ which is $\langle v, w\rangle=\operatorname{Re}\left(v^{\prime} \bar{w}\right)$. Observe that $D_{s} \tilde{T}(s, 1)$ is $\frac{d}{d s} \tilde{T}(s, 1)$ projected onto $H_{\tilde{\gamma}(s)}$. Since $\langle\mu, \dot{\tilde{\gamma}}(0)\rangle=0$,

$$
\left.\frac{d^{2}}{d s^{2}} d_{g}^{2}(\gamma(s), m)\right|_{s=0}=2\left\langle\left.\frac{d}{d s} \tilde{T}(s, 1)\right|_{s=0}, \dot{\tilde{\gamma}}(0)\right\rangle
$$

From (3.5) we have

$$
\begin{aligned}
\left.\frac{d}{d s} \tilde{T}(s, 1)\right|_{s=0} & =\left(\left.\frac{d}{d s}\left(\frac{\rho(s) \cos (\rho(s))}{\sin (\rho(s))}\right)\right|_{s=0}\right) \mu+\left(\left.\frac{\rho(s) \cos (\rho(s))}{\sin \rho(s)}\right|_{s=0}\right) \dot{\tilde{\gamma}}(0) \\
& -\left(\left.\frac{d}{d s}\left(\frac{\rho(s)}{\sin (\rho(s)) \cos (\rho(s))}\right)\right|_{s=0}\right)\left(\bar{z}^{\prime} \mu\right) z \\
& -\left(\left.\frac{\rho(s)}{\sin (\rho(s)) \cos (\rho(s))}\right|_{s=0}\right)\left(\bar{z}^{\prime} \dot{\tilde{\gamma}}(0)\right) z
\end{aligned}
$$

and along with (3.3), we get

$$
\left.\frac{d}{d s} \rho(s)\right|_{s=0}=\frac{-1}{\sin (r)}\left\langle\dot{\tilde{\gamma}}(0), \frac{\bar{z}^{\prime} \mu}{\cos (r)} z\right\rangle\left(r:=d_{g}\left(m, \mu_{I}\right)\right)
$$

Hence

$$
\begin{aligned}
\left\langle\left.\frac{d}{d s} \tilde{T}(s, 1)\right|_{s=0}, \dot{\tilde{\gamma}}(0)\right\rangle & =r \frac{\cos (r)}{\sin (r)}\|\dot{\tilde{\gamma}}(0)\|^{2}-\left(\frac{1}{\sin ^{2} r}-r \frac{\cos (r)}{\sin ^{3}(r)}\right)(\operatorname{Re} x)^{2} \\
& +\frac{r}{\sin (r) \cos (r)}(\operatorname{Im} x)^{2}
\end{aligned}
$$

where

$$
x=e^{i \theta} z^{\prime} \overline{\dot{\tilde{\gamma}}(0)}, e^{i \theta}=\frac{\bar{z}^{\prime} \mu}{\operatorname{cosr}} .
$$

The value of $x$ in (3.10), and hence the expression in (3.9), depends on $z$ only through $m=[z]$. Also if $\gamma=\pi\left(\gamma_{1}\right)=\pi\left(\gamma_{2}\right), \gamma_{1}$ and $\gamma_{2}$ being two geodesics on $S_{2}^{k}$ starting at $\mu_{1}$ and $\mu_{2}$ respectively, with $\left[\mu_{1}\right]=\left[\mu_{2}\right]=[\mu]$, then $\gamma_{1}(t)=\lambda \gamma_{2}(t)$, where $\mu_{2}=\lambda \mu_{1}, \lambda \in \mathbb{C}$. Now it is easy to check that the expression in (3.9) depends 
on $\mu$ only through $[\mu]=\mu_{I}$. Note that $|x|^{2}<1-\cos ^{2} r$. So when $|\dot{\gamma}(0)|=1,(3.9)$ is

$$
\begin{aligned}
& r \frac{\cos (r)}{\sin (r)}-\left(\frac{1}{\sin ^{2} r}-r \frac{\cos (r)}{\sin ^{3} r}\right)(\operatorname{Rex})^{2}+\frac{r}{\sin r \cos r}(\operatorname{Im} x)^{2} \\
> & r \frac{\cos (r)}{\sin (r)}-\left(\frac{1}{\sin ^{2} r}-r \frac{\cos (r)}{\sin ^{3} r}\right) \sin ^{2} r=\frac{2 r-\tan r}{\tan r},
\end{aligned}
$$

which is $>0$ if $r \leq R$ where $\tan (R)=2 R, R \in\left(0, \frac{\pi}{2}\right)$. Thus if $\operatorname{supp}(Q) \subseteq B\left(\mu_{I}, R\right)$, then $\left.\frac{d^{2}}{d s^{2}} d^{2}(\gamma(s), m)\right|_{s=0}>0$, and hence $\Lambda$ is positive definite.

Remark 3.1. It can be shown that $R \in\left(\frac{\pi}{3}, \frac{2 \pi}{5}\right)$. It is approximately $0.37101 \pi$.

Remark 3.2. The nonsingularity of $\Sigma$ defined in Theorem 2.2 is a mild condition which holds in particular if $Q$ has a density (component) with respect to the volume measure on $\Sigma_{2}^{k}$.

From Proposition 2.1, Theorem 3.1 and Remark 3.2, we conclude that if $\operatorname{supp}(Q)$ $\subseteq B\left(\mu_{I}, R\right)$ and if $\Sigma$ is nonsingular (e.g., if $Q$ is absolutely continuous), then the sample mean from an iid sample has an asymptotically normal distribution with nonsingular dispersion. To get the expressions for $\Sigma$ and $\Lambda$, note that the coordinate $\phi$ in Theorem 3.1 has the form

Hence

$$
\phi(m)=\left(\tilde{m}^{1}, \ldots, \tilde{m}^{k-2}\right)^{\prime}, \tilde{m}^{j}=\frac{r}{\operatorname{sinr}} e^{i \theta} \bar{v}_{j}^{\prime} z
$$

$$
\begin{aligned}
& \Sigma_{(2 k-4) \times(2 k-4)}=\left[\begin{array}{cc}
\Sigma_{11} & \Sigma_{12} \\
\Sigma_{12}^{\prime} & \Sigma_{22}
\end{array}\right], \\
& \left(\Sigma_{11}\right)_{i j}=4 E\left(\operatorname{Re}\left(\tilde{X}_{1}^{i}\right) \operatorname{Re}\left(\tilde{X}_{1}^{j}\right)\right),\left(\Sigma_{12}\right)_{i j}=4 E\left(\operatorname{Re}\left(\tilde{X}_{1}^{i}\right) \operatorname{Im}\left(\tilde{X}_{1}^{j}\right)\right), \\
& \left(\Sigma_{22}\right)_{i j}=4 E\left(\operatorname{Im}\left(\tilde{X}_{1}^{i}\right) \operatorname{Im}\left(\tilde{X}_{1}^{j}\right)\right), \quad 1 \leq i, j \leq k-2,
\end{aligned}
$$

and

$$
\Lambda_{(2 k-4) \times(2 k-4)}=\left[\begin{array}{cc}
\Lambda_{11} & \Lambda_{12} \\
\Lambda_{12}^{\prime} & \Lambda_{22}
\end{array}\right],
$$

where if $\dot{\tilde{\gamma}}(0)=\sum_{j=1}^{k-2} x^{j} v_{j}+\sum_{j=1}^{k-2} y^{j}\left(i v_{j}\right), x=\left[x^{1} \ldots x^{k-2}\right]^{\prime}, y=\left[y^{1} \ldots y^{k-2}\right]^{\prime}$, then

$$
\left.E\left(\frac{d^{2}}{d s^{2}} d_{g}^{2}\left(\gamma(s), X_{1}\right)\right)\right|_{s=0}=x^{\prime} \Lambda_{11} x+y^{\prime} \Lambda_{22} y+2 x^{\prime} \Lambda_{12} y
$$

This gives for $1 \leq r, s \leq k-2$,

$$
\begin{aligned}
&\left(\Lambda_{11}\right)_{r s}=2 E\left[d_{1} \cot \left(d_{1}\right) \delta_{r s}\right.-\frac{\left(1-d_{1} \cot \left(d_{1}\right)\right)}{d_{1}^{2}}\left(\operatorname{Re} \tilde{X}_{1}^{r}\right)\left(\operatorname{Re} \tilde{X}_{1}^{s}\right) \\
&\left.+\frac{\tan \left(d_{1}\right)}{d_{1}}\left(\operatorname{Im} \tilde{X}_{1}^{r}\right)\left(\operatorname{Im} \tilde{X}_{1}^{s}\right)\right], \\
&\left(\Lambda_{22}\right)_{r s}=2 E\left[d_{1} \cot \left(d_{1}\right) \delta_{r s}-\right. \frac{\left(1-d_{1} \cot \left(d_{1}\right)\right)}{d_{1}^{2}}\left(\operatorname{Im} \tilde{X}_{1}^{r}\right)\left(\operatorname{Im} \tilde{X}_{1}^{s}\right) \\
&\left.+\frac{\tan \left(d_{1}\right)}{d_{1}}\left(\operatorname{Re} \tilde{X}_{1}^{r}\right)\left(\operatorname{Re} \tilde{X}_{1}^{s}\right)\right], \\
&\left(\Lambda_{12}\right)_{r s}=-2 E\left[\frac{\left(1-d_{1} \cot \left(d_{1}\right)\right)}{d_{1}^{2}}\left(\operatorname{Re} \tilde{X}_{1}^{r}\right)\left(\operatorname{Im} \tilde{X}_{1}^{s}\right)+\frac{\tan \left(d_{1}\right)}{d_{1}}\left(\operatorname{Im} \tilde{X}_{1}^{r}\right)\left(\operatorname{Re} \tilde{X}_{1}^{s}\right)\right],
\end{aligned}
$$


where $d_{1}=d_{g}\left(X_{1}, \mu_{I}\right)$.

\section{ACKNOWLEDGEMENT}

The authors would like to thank the referee for the careful reading of the manuscript and for suggestions.

\section{REFERENCES}

[1] A. Bhattacharya and R. Bhattacharya, Nonparametric Statistics on Manifolds with Applications to Shape Spaces. In Pushing the Limits of Contemporary Statistics: Contributions in Honor of J. K. Ghosh, IMS Lecture Series (S. Ghoshal and B. Clarke, eds.), 2008.

[2] R. Bhattacharya and V. Patrangenaru, Large sample theory of intrinsic and extrinsic sample means on manifolds. I, Ann. Statist. 31 (2003), 1-29. MR1962498(2004a:60069)

[3] R. Bhattacharya and V. Patrangenaru, Large sample theory of intrinsic and extrinsic sample means on manifolds. II, Ann. Statist. 33 (2005), 1225-1259. MR2195634 (2007j:60020)

[4] F. L. Bookstein, Morphometric Tools for Landmark Data: Geometry and Biology, Cambridge Univ. Press (1991). Reprinted 1997. MR1469220 (99d:92003)

[5] M. P. do Carmo, Riemannian Geometry, Birkhäuser, Boston (1992). English translation by F. Flaherty. MR.1138207 (92i:53001)

[6] I. L. Dryden and K. V. Mardia, Statistical Shape Analysis, Wiley, Chichester (1998). MR1646114(2000b:60022)

[7] N. I. Fischer, T. Lewis and B. J. Embelton, Statistical Analysis of Spherical Data, Cambridge Univ. Press (1987). MR899958 (89b:62002)

[8] H. Hendriks and Z. Landsman, Mean location and sample mean location on manifolds: Asymptotics, tests, confidence regions, J. Multivariate Anal. 67 (1998), 227-243. MR 1659156 (2000a:62125)

[9] H. Karchar, Riemannian center of mass and mollifier smoothing, Comm. Pure Appl. Math. 30 (1977), 509-541. MR 0442975 (56:1350)

[10] J. Jost, Riemannian Geometry and Geometric Analysis, $4^{\text {th }}$ ed., Springer, Berlin (2005). MR2165400 (2006c:53002)

[11] D. G. Kendall, Shape manifolds, Procrustean metrics, and complex projective spaces, Bull. London Math. Soc. 16 (1984), 81-121. MR737237 (86g:52010)

[12] W. S. Kendall, Probability, convexity, and harmonic maps with small image. I. Uniqueness and fine existence, Proc. London Math. Soc. 61 (1990), 371-406. MR1063050 (91g:58062)

[13] H. Le, Locating Fréchet means with application to shape spaces, Adv. Appl. Prob. 33 (2001), 324-338. MR1842295 (2002d:60008)

[14] J. M. Lee, Riemannian Manifolds: An Introduction to Curvature, Springer-Verlag, New York (1997). MR1468735 (98d:53001)

[15] K. V. Mardia and V. Patrangenaru, Directions and projective shapes, Ann. Statist. 33 (2005), 1666-1669. MR2166559(2007a:62041)

[16] X. Pennec, Probabilities and statistics on Riemannian manifolds: Basic tools for geometric measurements, NSIP'99 (1999). MR2254442

Department of Mathematics, University of Arizona, Tucson, Arizona 85721

E-mail address: abhishek@math.arizona.edu

Department of Mathematics, University of Arizona, Tucson, Arizona 85721

E-mail address: rabi@math.arizona.edu 